\title{
Multiple Description Coding Based Network Coding
}

\author{
Hussein H. Maza'ar \\ Information Technology Dept. \\ Cairo University \\ Giza, Cairo 12613, Egypt
}

\author{
Hesham N. Elmahdy \\ Information Technology Dept. \\ Cairo University \\ Giza, Cairo 12613, Egypt.
}

\begin{abstract}
This paper presents a proposal for transmitting images using Multiple Description Coding (MDC) techniques over multiple links in a mesh network, while using network coding technique to add protection paths. In pure MDC, the losses of one or two of the multiple descriptions degrade the received image quality. In the proposed technique, the protection paths ensure that all the descriptions are received even when one or two descriptions are lost, but at the expense of additional links and data bandwidth. A comparative evaluation based on simulation and objective quality metrics is presented.
\end{abstract}

\section{General Terms}

Network Coding, , Mesh Network, Bilinear Interpolation.

\section{Keywords}

Network Coding, Quality Factor, Mesh Network, Bilinear Interpolation, Bite Rate, Polyphase Subsampling, PSNR.

\section{INTRODUCTION}

One of the main services of operation networks that must be deployed efficiently is reliability. In order to deploy a reliable networking strategy, one needs to protect the transmitted packets over unreliable links. Link failures are common problems that might occur frequently in single and multiple operating communication circuits. There is a wide variety of unintentional failures caused by natural disasters (earthquakes, fires, and floods), wear out, overload, software bugs, human errors, and so on, as well as intentional failures caused by maintenance action or sabotage. Such failures affect network facilities such as transmission or switching infrastructure, whose failure in turn disrupts communication services for business and residential users in Communications networks [10].

In network survivability and network resilience, one needs to design efficient strategies to overcome this dilemma [2]. Network coding is a powerful tool that has been recently used to increase the throughput, capacity, and performance of communication networks. Network coding is used to maximize the throughput. Also, it is robust against packet losses and network failures. As an application of network coding, data loss because of failures in communication links can be detected and recovered if the sources are allowed to perform network coding operations [2]. Network coding encodes/decodes the packets using XOR operations [1]. In order to deploy a reliable networking strategy, the transmitted data must be protected over unreliable links. The sender is able to combine their data into a single extra path (protection path) that is used to protect all data on the working paths carrying data from all sources. Also, there exist another solution to solve the packet loss over error-prone networks or unreliable links called Multiple Description Coding (MDC). Multiple Description Coding (MDC) is a source coding technique where the source is encoded into two or more descriptions. It has the capability of forming self decodable data packets"descriptions" [4]. If there is packet loss, the decoder uses the received descriptions to estimate the lost data. In the case of full packet reception, higher quality could be guaranteed [3]. The recent boom in multimedia applications triggered the interest in multiple descriptions; also the transmitting of high quality data is not a commodity but rendered a crucial issue, such as a large image block or speech block.

This paper is organized as follows. Section 2 mentions the related works of data recovery methods over networks and section 3 presents the network topology. In section 4, we present the idea of network coding and section 5 discussed the subsampling method.

In section 6, the classical multiple description image coding Methods are discussed shortly. Section 7 and section 8 present the proposed (MDC+NC) multiple description coding based network coding against single and two link failures. Section 9 discusses the simulation results and the paper is concluded in section 10 .

\section{THE RELATED WORK}

In [8] authors have proposed employing the network coding technique in order to protect against single link failure, in a manner that achieves both agility and resource efficiency. In [11], authors used the orthogonal and quincunx subsampling and JPEG2000 with directional lifting-based transform and their results are compared with the bicubic and texture orientation interpolation. In [9], author used the polyphase subsampling with JPEG standard to encode the descriptions. The author discussed the Wyner-Ziv transcoding and his results are compared with the bilinear interpolation. In [5], the author introduced a $1+\mathrm{N}$ protection model in optical mesh networks using network coding over p-cycles. The author suggested a model for protecting $\mathrm{N}$ connections from a set of sources to a set of receivers in a network with $\mathrm{n}$ connections, where one connection might fail. The suggested model can protect against a single link failure in any arbitrary path connecting a source and destination. In [6], the author extended the previous model to protect multiple link failures. It is shown that protecting against $\mathrm{m}$ failures, at least $\mathrm{m}$ p-cycles are needed. An illustrative example in case of two link failures was given. The idea was to derive $\mathrm{m}$ linearly 
independent equations to recover the data sent from $\mathrm{m}$ sources. In [7], the author extended the protection model in [9] and provided a GMPLS-based implementation of a link protection strategy that is a hybrid of $1+\mathrm{N}$ and $1: \mathrm{N}$. It is claimed that the hybrid $1+\mathrm{N}$ link protection provides protection at higher layers and with a speed that is comparable to the speed achieved by the physical layer implementations. In addition, it has less cost and much flexibility.

\section{NETWORK TOPOLOGY AND SETUP}

The mesh network depicted in Figure 1 is a mesh network. It is composed of one sender, multiple links and one terminal node (receiver). The $\mathrm{K}$ parameter in network equals the number of descriptions (links) which is determined by subsampling method.

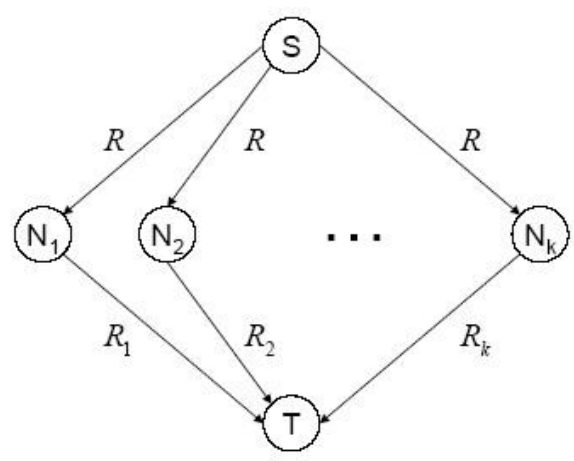

Figure 1: Two-hop Mesh Network.

\section{NETWORK CODING FUNDAMENTALS}

To illustrate the power of network coding, we provide a simple example, which is often referred to as the Wheatstone bridge, due to its electrical circuit's origin. It demonstrates that multicast routing can achieve the maximum possible throughput in a communication network using a coding scheme consisting of linear operations in finite field, whereas traditional store-andforward routing cannot achieve the same throughput. Consider the example shown in Figure 2(a), where the nodes $\mathrm{X}$ and $\mathrm{Y}$ respectively want to send two bits $b_{1}$ and $b_{2}$ to each other. One way of doing this is to let the bit $\mathrm{b}_{1}$ travel on the path $X \rightarrow A \rightarrow$ $B \rightarrow Y$ at one point of time and to let $\mathrm{b}_{2}$ travel on the path $Y \rightarrow A$ $\rightarrow B \rightarrow X$ on the other. However, if the network wants to transmit the bits simultaneously, then there is no way to do so, as there are no disjoint paths between $\mathrm{X}$ and $\mathrm{Y}$. However, using network coding as shown in Figure 2(b), one can save bandwidth. In this case, both $\mathrm{X}$ and $\mathrm{Y}$ transmit the bits $b_{1}$ and $b_{2}$ (as shown in the Figure 2) and then A XORs (encodes) them together and the resulting bit $b 1+b 2$ travels over the paths $A \rightarrow$ $B \rightarrow Y$ and $A \rightarrow B \rightarrow X$. Since node $\mathrm{X}$ already has of $b_{1}$, it can recover (decode) $b_{2}$ by the operation $b 1+(b 1+b 2)$. Similarly $\mathrm{Y}$ can also decode $b 1$.

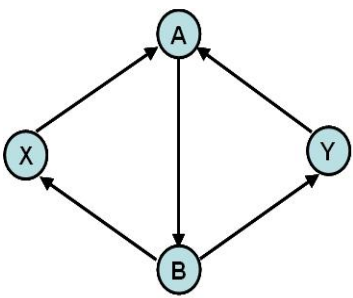

(a)

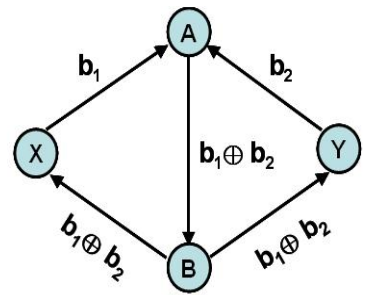

(b)
Figure 2: Network Coding on a Wheatstone bridge.

\section{THE SUBSAMPLING METHODS}

Polyphase subsampling is used in creating multiple descriptions of an image [3]. The results are displayed as descriptions as follows:

$$
\begin{aligned}
& X_{1}(i, j)=X(2 i, 2 j) \\
& X_{2}(i, j)=X(2 i+1,2 j+1) \\
& X_{3}(i, j)=X(2 i, 2 j+1) \\
& X_{4}(i, j)=X(2 i+1,2 j)
\end{aligned}
$$

Where, $X$ equals the original image. $X_{n}, n=1,2,3,4$ equals the sub-images or descriptions. The sub-images are encoded by baseline JPEG standard at certain quality factor. To prevent the original quality of image, we do not perform prefiltering before polyphase subsampling. Alternatively, smoothing kernels are applied at decoding operations [9].

\begin{tabular}{|l|l|l|l|l|l|l|l|}
\hline 1 & 3 & 1 & 3 & 1 & 3 & 1 & 3 \\
\hline 4 & 2 & 4 & 2 & 4 & 2 & 4 & 2 \\
\hline 1 & 3 & 1 & 3 & 1 & 3 & 1 & 3 \\
\hline 4 & 2 & 4 & 2 & 4 & 2 & 4 & 2 \\
\hline 1 & 3 & 1 & 3 & 1 & 3 & 1 & 3 \\
\hline 4 & 2 & 4 & 2 & 4 & 2 & 4 & 2 \\
\hline 1 & 3 & 1 & 3 & 1 & 3 & 1 & 3 \\
\hline 4 & 2 & 4 & 2 & 4 & 2 & 4 & 2 \\
\hline
\end{tabular}

Figure 3: Polyphase Subsampling.

\section{THE CLASSICAL MDC}

The traditional way of MDC with four descriptions and one central decoder is illustrated in Fig. 3. An input image $X$ is compressed by MDC into four descriptions of rates $R_{1}, R_{2}, R_{3}$ and $\mathrm{R}_{4}$. The central decoder retrieves the image at less distortion $\mathrm{R}_{1}, \mathrm{R}_{2}, \mathrm{R}_{3}$ and $\mathrm{R}_{4}$ are received. In case of two failures (i.e. two descriptions are lost), the image interpolation techniques are used to estimate the lost pixels at central decoder by neighboring pixels. 


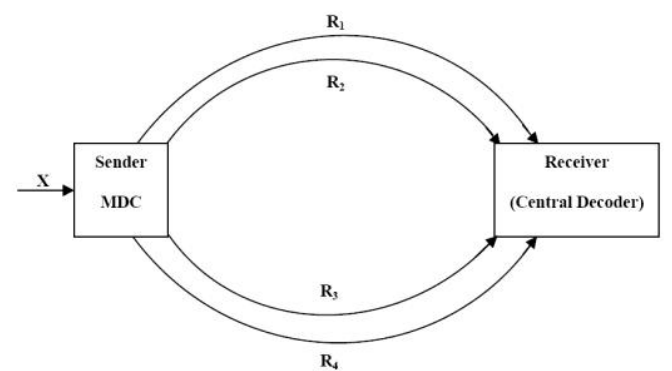

Figure 4: The typical scenario of MDC with 4 descriptions and central decoder.

\section{MDC BASED NETWORK CODING AGAINST SINGLE LINK FAILURE}

The goal of the image recovery strategy is to withhold rerouting the signals or the transmitted packets due to link failures over networks. However, we provide strategies that utilize network coding and reduced capacity at the source nodes. We assume that the source nodes are able to perform encoding operations and the receiver nodes are able to perform decoding operations. To decrease the distortion in central decoder when one of the descriptions is lost, we propose a method that can increase the quality of reconstruction and recover the lost data. We compress the image $\mathrm{X}$ using MDC and apply XOR operation on all compressed descriptions to deliver the data to the central decoder, if one of the descriptions is lost, the central decoder can recover the lost description via the protected path as in Equation 5 .

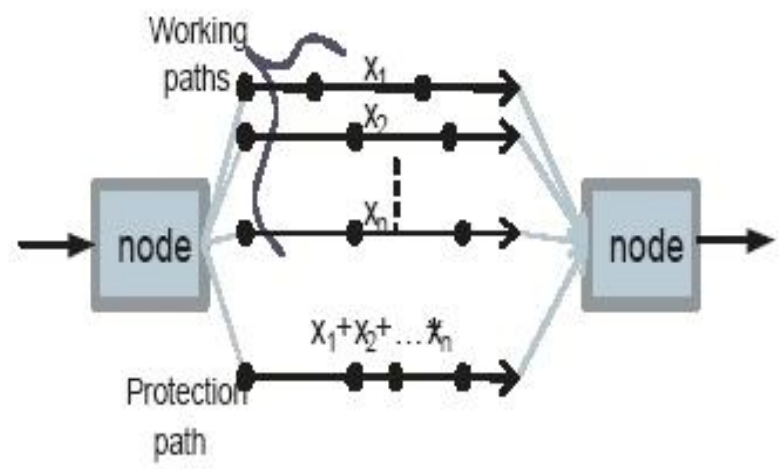

Figure 5: Network Protection against a Single Link Failure Using Network Coding

$$
\underbrace{x_{i}}_{\text {lostdesc }}=\sum_{j=1, j \neq i}^{4} x_{j} \oplus y
$$

where $y=\sum_{i=1}^{4} x_{i}$

\subsection{Encoding Operations}

The encoded operation of the proposed method against single lost description can be done as follows:

- The sender creates the descriptions and encodes each description using baseline JPEG standard with desired quality factor and assigns the descriptions to working paths (links).

- The protection path is achieved by bit-wise operation.

\section{Decoding Operations}

The decoding operations are analogous to the encoding operations. The terminal node (receiver) will receive the data from all working and protection paths and will do the decoding operations. In the case of single failure, there are the following possibilities:

- If the failed path is a working path, then the terminal node must query all other nodes in order to recover the failed data.

- If the failed path is the protection path, then no need to perform any action, since protected path is used for protection and does not have any valued data.

\section{MDC BASED NETWORK CODING AGAINST TWO LINK FAILURES}

The multiple links failures are found in some networks, so we will discuss the image recovery against two link failures with four connections. There are four links that carry the compressed data and two links carry the protected data. The location of failures is known and all operations are performed over finite field $\{0,1\}$.

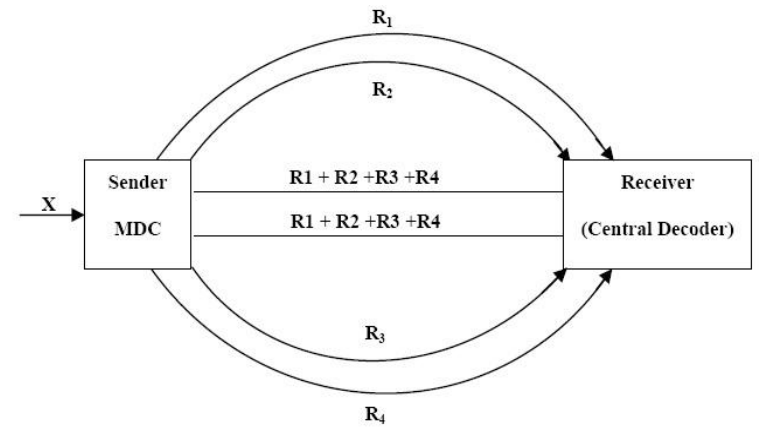

Figure 6: The Proposed (MDC+NC) with Four Descriptions and One Central Decoder ( Receiver ).

In the below scheme, there are four links carry the compressed data $\mathrm{R}_{1}, \mathrm{R}_{2}, \mathrm{R}_{3}$ and $\mathrm{R}_{4}$ and two links carry the protected data $\mathrm{y}_{1}$ and $y_{2}$. The two links are fixed and they are not chosen alternatively among the four links. In case of two link failure with polyphase sub sampling, the below scheme describes the model as follow: 


\begin{tabular}{|c|c|c|c|c|c|c|c|}
\hline Blocks & \multicolumn{6}{|c|}{ Block $_{1}$} & $\cdots$ \\
\hline Links & $L_{1}$ & $L_{2}$ & $L_{3}$ & $L_{4}$ & $L_{4+1}$ & $L_{4+2}$ & $\cdots$ \\
\hline $\operatorname{desc}_{1}$ & $R_{1}$ & O & $\mathrm{O}$ & O & $p_{11} R_{1}$ & $p_{12} R_{1}$ & $\cdots$ \\
\hline $\operatorname{desc}_{2}$ & 0 & $R_{2}$ & 0 & 0 & $p_{21} R_{2}$ & $p_{22} R_{2}$ & $\cdots$ \\
\hline $\operatorname{desc}_{3}$ & 0 & 0 & $R_{3}$ & 0 & $p_{31} R_{3}$ & $p_{32} R_{3}$ & $\cdots$ \\
\hline $\operatorname{desc}_{4}$ & 0 & 0 & 0 & $R_{4}$ & $p_{41} R_{4}$ & $p_{42} R_{4}$ & $\cdots$ \\
\hline total & $R_{1}$ & $R_{2}$ & $R_{3}$ & $R_{4}$ & $y_{1}$ & $y_{2}$ & $\cdots$ \\
\hline
\end{tabular}

All $\mathrm{y}_{\mathrm{j}}$ are defined as:

$$
y_{j}=\sum_{i=1}^{n} \alpha_{i j} R_{i}
$$

The coefficients are chosen over a finite field $\mathbf{F}_{\mathrm{q}}$ with $\mathrm{q}>\mathrm{n}-2$. One way to choose these coefficients is by using the follow two vectors.

$$
\left[\begin{array}{ccccc}
1 & 1 & 1 & \cdots & 1 \\
1 & \alpha & \alpha^{2} & \cdots & \alpha^{n-3}
\end{array}\right]
$$

We will provide two backup paths to protect against two disjoint links, which might experience failures. These two protection paths can be chosen using network provisioning. The protection paths are fixed for all Blocks. In the case of two failures, the central decoder will be able to solve two linearly independent equations in two unknown variables. For instance, assume the two failures occur in paths description one and two. Then the central decoder will be able to construct two equations with coefficients.

$$
\left[\begin{array}{ll}
1 & 1 \\
1 & \alpha
\end{array}\right]
$$

Therefore, we have

$$
\begin{aligned}
& \mathrm{R}_{1}+\mathrm{R}_{2}=0 \\
& \mathrm{R}_{1}+\alpha \mathrm{R}_{2}=0
\end{aligned}
$$

One can multiply the first equation by alpha and subtract the two equations to obtain value of $R_{2}$.

\subsection{Encoding Operations}

The encoded operation of the proposed method against two link failures can be done for each block as follows:

- The sender creates the descriptions and encodes each description using baseline JPEG standard with desired quality factor and assigns the descriptions to working paths (links).

- The protection paths are done by linear operation of sources coefficients which is the fastest arithmetic operation that can be performed among all sources data.

\subsection{Decoding Operations}

The decoding operation is analogous to the encoding operation. The terminal node will receive the data from all working and protection paths and will do the decoding operations. The objective of the decoding and recovery process is to withhold rerouting the signals or the transmitted packets due to link failures. In the case of two link failures, there are the following possibilities:

- If the failed paths are the protection paths, then no need to perform any action, since protected paths are used for protection and does not have any valued data.

- If the two link failures occur in two working paths, then in this case the two protection paths are used to recover the lost data. The idea of recovery in this case is to build a system of two equations with two unknown variables.

- If the two link failures occur in one protection path and one working path, then recovery operations are required. The receiver must recover its data using one of the protection paths.

\section{SIMULATION RESULTS}

Experiments are conducted and the results are presented in this section to evaluate the performance of the proposed and classical methods. The implementation is integrated into JPEG standard coding using the MTLAB R2008a. We show the results of polyphase subsampling with classical MDC based bilinear interpolation and proposed MDC based network coding. Firstly, the descriptions are created, and then we encode each description using the baseline JPEG standard with different quality factors. The sender combines their data into extra paths called protection paths that is used to protect all data on the working paths carrying data from all sources. To prevent blocking artifacts at low rate $\mathrm{R}$, the pixels of the received descriptions are smoothed using the filter kernel.

$$
\left(\begin{array}{ccc}
0.05 & 0.1 & 0.05 \\
0.1 & 0.4 & 0.1 \\
0.05 & 0.1 & 0.05
\end{array}\right)
$$

Three JPEG test images (Lena, bird and Baboon) of the resolution $256 * 256$ are used in our experiments. After the descriptions are received, we reconstruct the image from the received descriptions with one or two lost description using MDC based bilinear interpolation and proposed MDC based Network Coding. The proposed $(\mathrm{MDC}+\mathrm{NC})$ is able to recover the lost data exactly, so the results of the proposed (MDC+NC) method equals to MDC without failure. In the classical method, the bilinear interpolation uses the four neighboring pixels with weighted average to estimate the missed pixels.

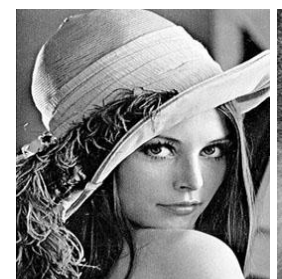

(a) Lena

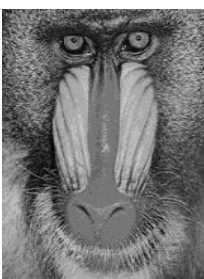

(b) baboon

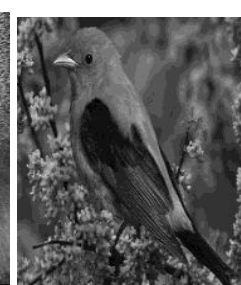

c) bird
Figure 7: Three Test Images. 
In table 1 and table 2, we compare the results of our experiments on test images using polyphase subsampling. We found that the proposed method $(\mathrm{MDC}+\mathrm{NC})$ improves the quality of image reconstruction rather than the classical method MDC based bilinear interpolation (MDC+BI). The values in table 1 and table 2 are PSNR against different quality factors $\{15,25,35,45,55$, $65,75$, and 85$\}$. Probably the most widely used objective measure is Peak Signal to Noise Ratio (PSNR). PSNR is measured on a logarithmic scale and is based on the mean squared error (MSE) between an original and decompressed image. Figures 8 and Figure 9 describe visually the values in Table 1 and Table 2.

\section{Table 1}

PSNR values of image reconstruction at receiver with single lost description using classical and proposed method and

Polyphase subsampling.

\begin{tabular}{|c|c|c|c|c|c|c|c|c|}
\hline \multirow{2}{*}{$\begin{array}{l}\text { PSNR (dB) } \\
\text { Quality Factor }\end{array}$} & \multicolumn{8}{|c|}{ Polyphase Subsampling } \\
\hline & 15 & 25 & 35 & 45 & 55 & 65 & 75 & 85 \\
\hline Classical (MDC+BI) - Lena & 29.49 & 30.01 & 30.38 & 30.61 & 30.75 & 30.90 & 31.15 & 31.44 \\
\hline Proposed (MDC+NC)- Lena & 30.58 & 31.01 & 31.25 & 31.52 & 31.72 & 31.96 & 32.28 & 32.67 \\
\hline Classical (MDC+BI) - baboon & 29.96 & 30.51 & 30.78 & 30.98 & 31.23 & 31.49 & 31.79 & 32.18 \\
\hline Proposed (MDC+NC) -baboon & 30.25 & 30.59 & 30.88 & 31.14 & 31.42 & 31.77 & 32.15 & 32.63 \\
\hline Classical (MDC+BI) - bird & 32.05 & 32.64 & 32.97 & 33.34 & 33.67 & 34.00 & 34.52 & 35.10 \\
\hline Proposed (MDC+NC) - bird & 32.10 & 32.67 & 33.03 & 33.46 & 33.80 & 34.23 & 34.87 & 35.61 \\
\hline
\end{tabular}

Table 2

PSNR values of image reconstruction at receiver with two lost descriptions using classical and proposed method and polyphase subsampling.

\begin{tabular}{|c|c|c|c|c|c|c|c|c|}
\hline PSNR (dB) & Polyph & se Subs & mpling & & & & & \\
\hline Quality Factor & 15 & 25 & 35 & 45 & 55 & 65 & 75 & 85 \\
\hline Classical (MDC+BI) - Lena & 30.50 & 30.81 & 31.00 & 31.19 & 31.30 & 31.46 & 31.66 & 31.88 \\
\hline Proposed (MDC+NC)- Lena & 30.58 & 31.01 & 31.25 & 31.52 & 31.72 & 31.96 & 32.28 & 32.67 \\
\hline Classical (MDC+BI) - baboon & 30.16 & 30.48 & 30.70 & 30.83 & 31.05 & 31.24 & 31.45 & 31.74 \\
\hline Proposed (MDC+NC) -baboon & 30.25 & 30.59 & 30.88 & 31.14 & 31.42 & 31.77 & 32.15 & 32.63 \\
\hline Classical (MDC+BI) - bird & 31.96 & 32.57 & 32.86 & 33.15 & 33.46 & 33.71 & 34.14 & 34.58 \\
\hline Proposed (MDC+NC) - bird & 32.10 & 32.67 & 33.03 & 33.46 & 33.80 & 34.23 & 34.87 & 35.61 \\
\hline
\end{tabular}




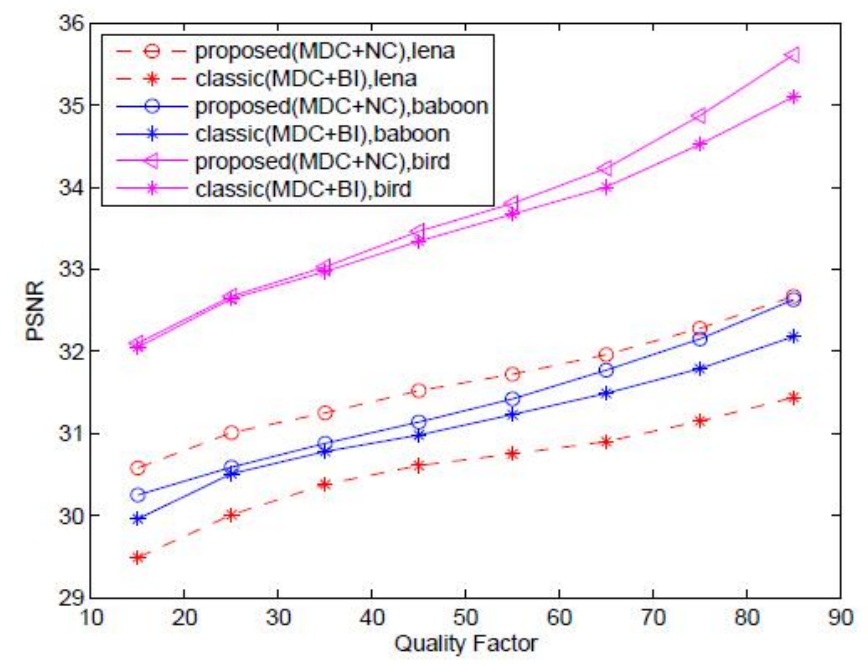

Figure 8: PSNR vs. Quality Factor of JPEG Reconstruction using MDC+BI and MDC+NC based Polyphase Subsampling against Single link Failure.

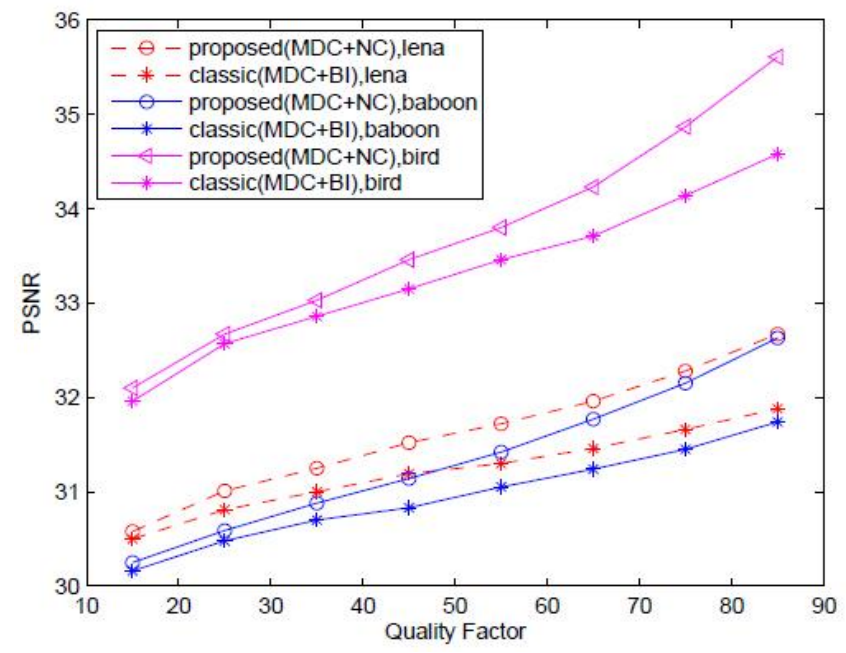

Figure 9: PSNR vs. Quality Factor of JPEG Reconstruction using MDC+BI and MDC+NC based Polyphase Subsampling against Two link Failures.

\section{CONCLUSION}

We introduced a proposed method called multiple description coding based network coding $(\mathrm{MDC}+\mathrm{NC})$ in mesh networks against single lost description. The proposed method outperforms better quality image reconstruction rather than other existing methods. We use the bilinear interpolation in classical method to estimate the lost description against the single failure and two failures. Our proposed method is useful in medical applications when the quality of image is important. This method has disadvantage because the cost of additional link. We apply these methods on mesh networks with one sender, multiple links and one terminal node (receiver). Furthermore, one can use this method to encode and decode the descriptions with different factors, rates and network models. We plan to investigate this method on JPEG 2000, different networks models and network video coding to improve the quality of videos and reduce delay at receivers. Also, we will discuss the proposed method (MDC+NC) against multiple lost descriptions. In a future research, the network coding will be applied on different networks to recover the data and overcome the problems of delay that produced from routing and retransmission mechanics.

\section{REFERNCES}

[1] S. A. Aly and A. E. Kamal. Network protection codes against link failures using network coding. In Proc. of IEEE 2008 Global Telecommunications Conference, Dec. 2008.

[2] S. A. Aly and A. E. Kamal. Network protection schemes for two link failures in optical networks. In Proc. of the 18th International Conference on Computer Theory and Applications (ICCTA'08), Alexandria, EG, October 11-13 2008.

[3] C. Ates, Y. Urgun, B. Demir, O. Urhan, and S. Erturk. Polyphase downsampling based multiple description images coding using optimal filtering with flexible redundancy insertion. In Proc. of International Conference on Signals and Electronic Systems (ICSES'08), Sept. 2008.

[4] V.K. Goyal. Multiple description coding: compression meets the network. IEEE Signal Processing Magazine, 18(5):7493, Sep 2001.

[5] A. Kamal. 1+n protection in mesh networks using network coding over p-cycles. In Proc. of IEEE Global Telecommunications Conference, 27 Dec. 2006.

[6] A. Kamal. $1+n$ protection against multiple link failures in mesh networks. In Proc. of IEEE International Conference on Communications, June 2007.

[7] A. Kamal. Gmpls-based hybrid 1+n link protection over pcycles: Design and performance. In Proc. of IEEE Global Telecommunications Conference, Nov. 2007.

[8] H. H. Maza'ar and H. N. Elmahdy. Multiple description image networks coding against single link failure in mesh networks. In Proc. of IEEE International Conference on Internet Multimedia Systems Architecture and Application, Bangalore, India, 122009.

[9] D. Varodayan, D. Chen, and B. Girod. Network image coding for multicast. In Proc. of IEEE 10th Workshop on Multimedia Signal Processing, Oct. 2008.

[10] M. Vasseur, J.and Pickavet and P. Demeester. Network Recovery: Protection and Restoration of Optical, SONETSDH, IP, and MPLS. Morgan Kaufmann, San Francisco, 2004.

[11] Nan Zhang, Yan Lu, Feng Wu, Xiaolin Wu, and Baocai Yin. Efficient multiple-description image coding using directional lifting-based transform. IEEE Transactions on Circuits and Systems for Video Technology, 18(5):646-656, May 2008. 